\title{
NEW ASPECTS IN GROUPING TYPICAL INJURIES FROM ROAD TRAFFIC ACCIDENTS
}

\author{
Petra Aradi ${ }^{1}$, Miklós Szokoly ${ }^{2}$ \\ ${ }^{1}$ Budapest University of Technology and Economics \\ 2 Péterfy S. Hospital and Trauma Center \\ petra.aradi@gmail.com
}

\begin{abstract}
Typical mechanisms and specific injuries of people involved in road traffic accidents are presented. Injury patterns specific to occupants of vehicles with enclosed passenger compartment, as well as unprotected participants of traffic (pedestrians, bicyclists, motorcyclists, etc.) are grouped.

Injury severity classification, treatment planning and outcome prediction is usually done based on various scoring systems, both internationally and in Hungary. The enormous number of such scoring systems make a thorough survey difficult and conversion among these scores has limitations. However prehospital care providers and hospital emergency staff need a "common language”, preferably a system that utilizes the advantages of scoring systems. There is no uniform practice of this communication and data transfer in Hungary now, that is why part of the important data from the incident scene might not get to the hospital together with the patient.
\end{abstract}

The documentation of prehospital care providers both in Hungary and abroad are discussed and analyzed. Score systems in prehospital and emergency medicine, as well as outcome prediction measures are covered. Data collection schemes especially the Utstein-Style for documenting major trauma and the German MIND2 (Minimale Notarztdatensatz, minimal prehospital care data set) are also presented.

A suggestion is introduced for the data content of prehospital documentation, so that it could further help hospital admission and care. The main aspects of the suggestion are road traffic accidents, because the creation of such a widely accepted and used document (a prehospital patient report form) requires a team of experts from various - mainly medical - specialties. Technical aspects, such as digital data collection are also covered. Future directions of development are named, too.

Keywords: road traffic accidents; prehospital care; emergency medicine; scoring systems; patient report form

\section{Introduction}

Accidents are an epidemic in our fast-paced, motorized era. Injuries and the consequent and definitive loss of health are an enormous financial and political problem for societies all over the world.
Road traffic accidents become more and more common, that is why various efforts are made to reduce their number and severity. Injury mechanisms, typical patterns and severity could be examined and relationships defined. These analyses and statistics might further help to fine treatment protocols, so that the 
best possible ways are used throughout patient care (on-site prehospital treatment, hospital admission and definitive medical attendance, and later on rehabilitation) to provide best outcome.

Communication between prehospital care providers and admitting hospitals needs an upgrade in aspects of transmitted patient data, as well as circumstances and characteristics of the accident.

A brief overview of road traffic accidents and injury mechanisms specific to such events is given. Prehospital care, documentation requirements, scoring systems and data collection schemes are discussed and analyzed, to help consider a new prehospital patient report form with modern data content, processability and ease of use.

\section{Road Traffic Accidents}

Road traffic accidents (RTA) are as old as mankind. The severity of injuries from RTAs is in proportion to the "development level" of vehicles. So long as people walked, it was easier to avoid a traffic accident and the consequences of accidents were milder, compared to the emergence of vehicles. The introduction of animal power, and later on mechanization significantly increased accident and injury risks. Spread of motorization together with increase of people's need/urge to locomotion caused significant changes is traffic habits. The rise of speed and the congestion of roads resulted in enormous growth of traffic accident occurrences. In the beginning of the 1960s US President John F. Kennedy said, that "traffic accidents are one of the greatest, perhaps the greatest of the nation's public health problems".

RTAs are responsible for the death of at least 1.3 million people yearly, the number of injured is at least 50 million. The majority of injured and dead are pedestrians. More the $85 \%$ of victims (and $96 \%$ of dead children) is a citizen of low- and middle-income countries. ${ }^{1}$ WHO (World Health Organization) forecasts traffic accidents to be the primary cause of premature death and permanent impairment of children of 4 years and older by 2015. This "hidden epidemic" is going to cause a crisis in the public health system. Predictions suggest today's 1.3 million RTA deaths per year to reach 1.9 million by 2020. Commission for Global Road Safety claims that global, regional and national development of public road traffic could prevent 5 million deaths and 50 million serious injuries in a year worldwide by 2020 .

\section{Injuries Related to Road Traffic Accidents}

Human body is a complex system of tissues with various mechanical properties. Damage resulting from impacts of certain magnitude, direction and duration on the human body depend on properties such as elasticity and resistance of individual tissues and organs. Beside general governing laws there is a huge effect of individual variations, that are partly consequences of inherited factors, are acquired like illnesses, however the role of age, general physical and mental status could neither be neglected. When a certain body part is injured, tissues with different properties get different damages.

During impact there is a significant amount of energy transferred to the injured person in a usually very short time. The amount of energy transferred could be estimated in view of the mechanism and circumstances of injury. In road traffic accidents the speed of the vehicle at the time of impact is in direct connection with the vehicle's kinetic energy. Vehicle's speed at impact could be estimated from deformations 
of vehicle body. Statistics show that a deformation greater than $35 \mathrm{~cm}$ usually cause serious injuries, a deformation over $50 \mathrm{~cm}$ is usually lethal for the person involved. These data are not universal, just an estimated average, because vehicle type, construction, mass and materials vary largely.

Kinetic energy transferred to the person appears as a shock wave at contact. Energy transfer produces cavitation, shock wave is transmitted by particles in tissues and hollow organs. Cavitation is either temporary or permanent. Energy transfer depends on the amount of particles involved, composition of bodies in contact, and area of contact that is connected to the shape and position of the object. Typical examples are lungs filled with compressible air, the gastrointestinal system partly filled by air, vascular system and bladder filled with non-compressible liquid, liver, spleen, kidneys and muscles partly filled by liquid, as well as solids in bones, road surface, steel, etc.

Energy transfer might induce blunt or penetrating injuries. Blunt injuries do not penetrate tissues, cavity is generated farther from the impact site, in impact direction. Penetrating injuries as the name suggests break through tissues, cavity is perpendicular to the direction of the penetrating object that damages tissues getting into its way.

Some injuries are classified as open wounds of mechanical origin ranging from simple abrasions to cuts, stabs, conquassations, often in a combined form. When there is a fire, burn of different severity might appear on the body surface and inside airways. Smoke and chemical inhalation are a risk too. Electrical injuries might also cause burns and life threatening heart rhythm irregularities. Mechanical effects might lead to injuries and tissue damages without visible bleeding. Closed injuries range from relatively simple suffusions, to more serious haematomas under the skin or inside the skull. Blunt forces might cause contusion, commotion, compression or crush, multiple fractures, nerve and spinal lesions. Special soft tissue damage is the usually closed decollement, its more serious form is however an open injury (degloving). It is usually the result of the body or extremities getting stuck between the road surface and the relatively elastic tire moving tangentially. Compartment syndrome arises when tissue pressure increases in muscle compartments of extremities. Typical injury patterns of inner organs are contusion, commotion and rupture, especially of liver, spleen, lungs and kidneys, and perforation of stomach, intestines and bladder. Secondary ruptures occur sometimes days after the accident leading to a serious life-threatening situation. In body cavities (skull, thorax, pleural cavity, pericardium, mediastinum and different parts of the abdominal cavity in relation with the peritoneum) not only blood, but air might cause space-occupying lesions, that compress organs in the affected body cavity. Fractures are bone injuries when the continuity of bones gets broken. Fractures can have both internal and external origins. Latter are magnitude, direction, duration and speed of increase of force causing the fracture. Internal aspects are the bone's energy dissipation capacity, elasticity, density, and resistance against fatigue breaks. Dangers of fractures are severe blood loss, nerve- and vascular injuries and infection. Fractures have various classifications available, e.g. dislocated or not, closed or open, number of broken parts, shape of the fracture, amount of collateral soft tissue damage, etc. Injuries of joints connecting bones range from contusion, and distorsion to luxation. Vascular injuries occur when the continuity of blood vessels is damaged, and result in bleeding. Three main groups of them are direct and indirect vascular injuries, and chronic damages as consequences of traumatic injuries. 
Oedema appear usually at the site of and near to the injury. Such space-occupying processes in the skull are life threatening, because intracranial pressure increases rapidly.

Injuries can be classified based on their number and extent. Monotrauma is the injury of one body part, multitrauma is multiple injuries. Multiple injuries apply to polytrauma too, however polytrauma has qualitative attributes compared to quantitative. According to Tscherne polytrauma is the simultaneous injuries of multiple body parts or organ systems, among which at least one injury or a combination of injuries is life-threatening. Most polytraumatised patients have serious injuries of body cavities (skull, thorax, abdomen $)^{2}$. Ahnefeld's extension is also valid in prehospital and emergency medicine, namely certain life-threatening monotrauma (isolated intracranial or severe thoracic injuries) has to be treated as polytrauma, because other, on the scene invisible injuries can not be excluded with absolute certainty ${ }^{3}$. Authors of a recent study categorized polytraumatised as patients suffering such (mostly) blunt injuries of multiple body regions or organs, that disturb normal function and consequently lead to functional disturbances in other, non-injured organs. Morbidity and mortality is higher in polytraumatised than the summed morbidity and mortality of their individual injuries. Major cause of death after polytraumatisation is the vicious circle of the lethal triad of hypothermia, coagulopathy and acidosis.

In traffic accidents forces proportional to the rate of speed change, namely acceleration or deceleration, the mass of vehicle and injured people cause deformation not only in the vehicle, but might lead to injuries of various severity (in extreme lethal) in people. The reason why injuries occur are either directly or indirectly the increase in kinetic energy. The rise in speed and kinetic energy are responsible for increased breaking distance, time to avoid the accident, magnitude of destructive forces, severity of injuries and probability of death. Kinetic energy is proportional to the square of velocity, the faster the speed, the shorter the reaction time, distances and time of effects of forces. A small difference in speed is a huge distinction in injury severity.

The spread and development of motorization result in new types and combinations of injuries in road traffic accidents, the number of polytraumatised grows. Typical injuries have to be graded to help prehospital care providers choose the appropriate facility, where treatment is organized along specific protocols, to safely predict outcome, as well as to serve scientific data collection, processing and comparison.

The knowledge on the course of the accident supports diagnostics (and later on therapy), because the knowledge of typical injury mechanisms help to look for or exclude damage specifically. In oxyology (prehospital emergency medicine) victims of road traffic accidents belong to either the protected or unprotected groups ${ }^{3}$. Protected are drivers and passengers of vehicles with enclosed passenger compartment, that shields occupant from direct impact. Unprotected participants of traffic (pedestrians, bicyclists, motorcyclists, etc.) are covered only by their garments.

It is obvious, that similar mechanisms cause more severe injuries in unprotected participants of traffic, and polytrauma is more frequent among them, than among members of the protected group. The evaluation of WHO underpins this thesis, it lists children, pedestrians, bicyclists and elderly as the most vulnerable.

In a road traffic accident the vehicle usually slows rapidly down before stopping, while people inside it continue to move with their original speed, because of their inertia. Forces 
acting upon occupants are proportional to change of speed, and their direction is governed by the area of collision. During collision driver and passengers suffer injuries in more than one phases. During primary collision vehicle's speed changes suddenly, however occupants' speed does not. Passive and active protection (safety belts, airbags, etc.) decrease occupants' original speed, however when they are not present or not applied, then parts of the vehicle body that the occupant hits take up their role. The sudden change of speed has different effects on body parts and tissues of various physical properties and attachments. The effect of impact and injuries depend upon, whether the occupant was the driver or a front seat passenger or someone sitting in the back row, as well as s/he perceived the accident situation in time and whether s/he was able to protect her/himself as a reflex action or voluntarily, or by chance s/he was asleep with toneless muscles.

Sudden deceleration makes lower limbs with tightened muscles hit the dashboard (front seat occupants), dashboard and steering column (driver), or the back of front row seats (back seat passengers). Contact areas suffer soft tissue damage, tightened muscles may lead to knee, thighbone and pelvic injuries, pedals are responsible for typical injuries, too. Body's inertia causes the head to swing forward, hit some part of the vehicle with resulting facial and head injuries, while cervical spine is injured by hyperflexion. Next the thorax dashes forward, resulting in extensive bone- and soft-tissue injuries. Cervical spine hyperextends and typical whiplash injury arises. The next phase is the repeated forward motion of head and torso, aggravating head, abdominal, thoracic and extremity injuries.

Organs suspended in body cavities and the brain in the hard skull boost forward from the sudden deceleration, than bang back.
Suspension system gets damaged, and organs are injured because of the repeated hits. Localized brain oedema appears below the place of impact (coup) or farther from it (contre-coup).

The driver is usually in a more favorable situation than the front seat passenger, because s/he usually perceives the situation sooner, and may better prepare to avert it. Head and chest of back seat passengers hit the upholstered seat back, causing significantly milder injuries than dashboard, windscreen, steering wheel and column A. When the head of an unrestrained ack seat passenger hits the head of a restrained front seat passenger, both suffer severe head trauma. Typical injuries of back seat passengers are severe pelvic injuries, fractures and dislocated hip. When front seat occupants not properly use safety belts or do not use at all, their injuries are more complicated, especially when airbags inflate.

In case of a rollover accident unrestrained occupants fall out, suffering further severe, often life-threatening injuries. When the occupant gets stuck under or inside the vehicle s/he might suffer crush injuries. By side impacts typical glass shard injuries of face, shoulder and arm occur.

Generally speaking motor vehicle occupants suffer injuries in three levels: head, thorax and abdomen, and knee-thigh-hip region.

Pedestrian accidents were analyzed in connection with hit speed, and a significant relationship was proven between hit speed and injury severity. Over $40 \mathrm{~km} / \mathrm{h}$ life-threatening injuries are expected, over $60 \mathrm{~km} / \mathrm{h}$ death is almost imminent. Beside hit speed, there are a number of other factors to concern, too. Probably the most important is the pedestrian's age, and in tight connection with it the state of the skeletal system, its rigidity and elasticity, the even- 
tual osteoporosis, and the frequency of injury complications. Injury severity depends not just on the magnitude, but the duration of impact. There is a significant difference in speed and mass and physical properties of pedestrian and vehicle, kinetic energy could be enormous. Pedestrian's "deformation" is highly complex, and it has three phases: pedestrian acquires the vehicle's speed when contact is made, gaining a huge amount of extra kinetic energy, then usually the body separates from the vehicle and after an airborne period crashes into the ground and skids or rolls until stop, losing the kinetic energy. The third phase is occasional, the pedestrian might crash into an other object or is hit or run over again. Pedestrian hits happen in a few seconds, that is why victims do not have enough time to react and try to avoid the accident.

The mechanism of total frontal hit is described by Waddell's triad: thighbone fracture, intrathoracic or intraabdominal injuries and head trauma on the opposite side.

Bicyclists suffer their injuries when they tip over, brake suddenly, or when a motor vehicle hits them. Different mechanisms might appear in combination, but magnitude and direction of impact force and change of speed is significant to each of those. Primary collision is often with a part of the bicycle, transmitting the force indirectly to the bicyclist. When the collision is is from the back, the bicycle accelerates, its driver falls to the motor vehicle or the ground and suffers injuries. The bike tumbles upon its rider causing further extremity injuries. Handlebar and its equipment, brakes and levers hurt hands. Handlebars might also cause severe abdominal injuries. Saddle and frame cause typical injuries in the perineal region in cyclists riding the bike. When a car overtaking the bicycle hits the biker from the left, the left lower extremity is injured and secondary injuries occur on the cyclist's other (right) side. When a bike turns in front of a motor vehicle its rider suffers similar injuries, however secondary injuries are on the same sides as the primary, because the biker's body contacts the front side and bonnet of the vehicle.

The speed of motorcyclists is comparable to the speed of cars and trucks, however the probability of death is almost 30 times that of occupants of a car. Size and mass of motorcyclists is much less, and their garment provide less protection than body and safety devices of a vehicle. Compared to pedestrians, bicycles and motorcycles with their riders on are unstable systems. Motorcycles are sometimes orders of magnitude heavier, more powerful and harder to control than bikes. Injury patterns are similar to those of bicyclists, but knee, elbow and shoulder injuries are frequent. Lower limb degloving injuries and conquassations, severe fractures are common, often resulting in the loss of the limb. Head injuries are also common, however safety helmets helped reduce the number and severity of head injuries since their wide-spread introduction. Thoracic injuries are also prevalent. Burns from the hot parts of the motorcycle are also common.

\section{Prehospital Care}

Prehospital care is organized around different schemes worldwide. These schemes integrate local rescue and ambulance systems and health network in a various scale. In Europe rescue services and ambulance services are usually clearly separated. The so-called Franco-German model operates with highly skilled doctors (in Hungary oxyologists and other specialists) on site. In Anglo-Saxon systems prehospital care is provided by well educated paramedics, who work by protocols with equipment appropriate to their skills. The 
basis of emergency care is the system of emergency departments and trauma centers. However simultaneous existence of personal and objective terms are needed in both systems. The main philosophical difference between these to systems is "scoop and run" and "stay and play". Today both principles are applied in a locally fitting proportion in a large number of countries.

In a trauma scene injury severity can not always be determined because of circumstances (e.g. jammed victim), the relatively spare diagnostic apparatus (e.g. no on site imaging and laboratory) and the shortness of time available. Severe injuries are presumed, based on the suspected injury mechanism determined by anamnesis and a quick look at the scene, significant changes in basic vital functions, injury patterns. Today Dr. R Adams Cowley's "golden hour of shock" refers rather to urgency and emergency in the care of severely injured, and not the strictly $60 \mathrm{~min}$ utes. Principles and educational systems of ATLS® (Advanced Trauma Life Support ${ }^{\circledR}$ for in hospital providers) and ITLS (International Trauma Life Support for on site providers) are accepted and applied in a large number of countries in the world. Checklists help prehospital providers worldwide to choose the appropriate treatment strategy based on quick and accurate decisions. Scoring systems are integral parts of some checklists.

The Methodological Guide of the Hungarian National Ambulance Service (Országos Mentôszolgálat, OMSZ) also refers to the mechanism of the event leading to the injury as a measure of injury severity. ${ }^{6}$ Injury mechanism informs prehospital providers whether a high energy, full body impact (run over, fall from height, frontal collision, fall from a vehicle), or the isolated injury of one or more body parts occurred, as well as about priority of transport or on site primary care.
In traffic accidents severe injury has to be assumed, when the pedestrian or bicyclist is run over or hit the ground, was dragged, high speed motorcycle or car accident happened (at least $50 \mathrm{~km} / \mathrm{h}$ with safety belt, at least $30 \mathrm{~km} / \mathrm{h}$ without safety belt or motorcycle, at least $5 \mathrm{~km} / \mathrm{h}$ by bicyclists), falling or flying out of a vehicle, falling from a vehicle in motion, turnover of a vehicle, victim is jammed (rescue can take long and delays definitive care), indirect signs (severely damaged passenger compartment, engine crushing into passenger compartment, serious frontal axle damage, inflated airbag, death in the same passenger compartment), fall from height (over $3 \mathrm{~m}$ ), explosion. ${ }^{6}$ A quick examination can also reveal severe injuries: unstable thorax, fracture of two or more long bones and/or pelvic fracture, high limb amputation, penetrating abdominal, thoracic, pelvic, neck or skull injury, smoke inhalation and at least $15 \%$ of body surface with $2^{\text {nd }}$ - or $3^{\text {rd }}$-degree burns. ${ }^{3}$

\section{Scoring Systems}

Scoring systems are used worldwide to assign one or more numbers to injury or illness severity. There are three basic groups of scoring systems: anatomical, physiological and outcome based, however in practice these categories can not be clearly separated. Creating numeric descriptors has more than one goals: the quick handover of information on the patient's status and the change of status during medical attendance between prehospital providers and hospital staff helps choosing appropriate treatment schemes (protocols), and later on it can be used in a scientific study that uses statistics. Scoring systems have to be simple, quick, and should support quality of treatment and outcome.

Today's trauma scoring systems together with anatomical and physiological scales help outcome prediction. One application area is pre- 
hospital triage, however quality control and assurance of groups of injured people, organization and development of trauma care systems also appear useful.

In the prehospital environment scoring systems are primarily patient's assessment, measurement and control of diagnostic and therapeutic efforts, outcome prediction and decision support in triage and therapy. Prehospital and emergency department scoring systems need to be based on such general vital parameters, that might be obtained throughout the whole system of care.

When a scoring system is applied, the patient's complex and diverse data have to be compiled into one or some representative severity measures (numbers). Scoring always means information loss. Efforts for more and more exact mappings resulted in an enormous number of scoring system with advantages and disadvantages. Inaccuracy in mapping is partly because of patients' different anatomical and physiological characteristics. Exact outcome prediction (Eq. 1.) must refer to earlier medical problems, that adversely affect patients' healing reserves.

$$
\begin{array}{r}
\text { outcome }=\text { function } \\
\text { (anatomical injuries, } \\
\text { physiological injuries, } \\
\text { patient reserves) }
\end{array}
$$

MedAL (The Medical Algorithms Project) chapter 29 "Trauma \& Emergency Medicine" has 59 topics with 562 scoring systems. ${ }^{7}$ Chapter 38 "Forensic Medicine" has 44 topics, among which only one ("Evaluation of Transportation Accidents") has only one traffic accident related scoring system ("Findings That May Identify the Driver in an Automobile or Pilot in an Airplane Accident"). ${ }^{7}$

Everyday practice uses a lot less scoring systems. Prehospital trauma scoring systems have to safely identify high- and low-risk patients, have high face-validity and inter-rater reliability, be easy to use, allow quick and accurate measurements. These systems are mostly physiological, while anatomical systems are good for forecasting mortality and hospital care, as well as post-processing. The most widely applied physiological scoring systems have a minimal instrument need, could be quickly determined, supporting triage and repeated retriage.

Glasgow Coma Scale (GCS) was developed to check people with head injury. GCS 13 corresponds to minor brain injury, GCS 9-12 is a moderate, GCS 8 or less corresponds to severe brain injury. GCS is the sum of three values, eye opening (1-4 points), verbal response (1-5 points) and motor response (1-6 points), ranging from 3 to 15 points, the more points representing better condition, however individual components are noted as well. GCS is widely used both in prehospital environment and in hospitals. GCS 8 or less is indication for assisted airway (endotracheal intubation and mechanical ventilation in most cases). Hospital admission GCS has one of the most important prognostic values, however it is to be evaluated only with additional neurological examinations. A change of 2 or more in GCS points means a significant change in the patient's status.

Revised Trauma Score (RTS) uses three parameters, respiratory rate, systolic blood pressure and the sum of GCS points. All three parameters can have $0-4$ points (worst-best), the sum of which is the RTS value. RTS of 11 or less indicates transport to a trauma center. RTS is hard to determine when exact GCS and spontaneous respiratory rate is not available, because the patient is intubated or is under medications, alcohol or drugs.

Effects of injuries depend on many parameters, quantity of tissue damage, physiological responses to the injury and other aspects, such 
as age, physical status, etc.. Tissue damage can be classified with the anatomical Abbreviated Injury Scale (AIS) and its descendants. ICD (International Classification of Diseases) based systems help in outcome prediction. There are more detailed scoring systems for organs and organ systems, such as AAST (American Association for the Surgery of Trauma) and AO/ OTA (Arbeitsgemeinschaft für Osteosynthesefragen, Orthopaedic Trauma Association) scales.

Abbreviated Injury Scale (AIS) was created in 1969 by American Medical Association (AMA), Society of Automotive Engineers and Association for the Advancement of Automotive Medicine (AAAM). Since the publication of the first AIS version, the International Injury Scaling Committee (IISC) organizes its development. AIS uses a severity score of 1-6 (light to severe) for seven body regions, with 9 as the measure of unknown severity. Injury Severity Score (ISS) is also an anatomical scoring system, that uses AIS severity points (1-6) and it is used to classify people with multiple injuries. ISS uses 6 body regions and has a disadvantage of considering only one injury per body region. New Injury Severity Score (NISS) corrected this problem, more than one serious injury in the same body region can be taken into account.

\section{Data Collection Schemes}

Quality assurance and documentation are strongly connected, the first provide that the latter maintains its function, documentation serves the basis of quality assurance. Until the end o the $20^{\text {th }}$ century there was no general demand to prepare fact-based studies on quality and efficiency of prehospital care.

In Hungary ambulance care documentation is regulated by law. Currently there are two gen- erally used documents of the National Ambulance Service and the volunteer Ambulance Service of the Hungarian Maltese Charity Service. One of the documents used by both organizations is the so-called log sheet, that contains mostly technical details (name, address of the patient and the description of the problem, times, distances, materials used). Prehospital care is documented in a prehospital patient report form. Legal regulations govern the handling and keeping of these (and other connected) documents.

To strengthen the link between prehospital and institutional care, new uniform administration schemes should be used to provide hospitals with every possible details of the circumstances of the injury, as well as details of prehospital and in-transport care. This link is supposed to provide and develop professional standards of patient care, and could help clearing legal aspects (e.g. complaints).

The prehospital patient report form of OMSZ is an A5 sized paper with both sides to fill (Figure 1). Data content is not very extensive, the form has to be filled with handwriting and there is not much help in the form of checklists and drawings. The only scoring system used is GCS. The main characteristics of this form is written information.

Figure 2 shows the prehospital patient report form of the Ambulance Service of the Hungarian Maltese Charity Service, that provides a completely volunteer, but professional highlevel ambulance 4 days a week in Budapest. This single A4 sized form is more user-friendly from the point of filling out, quite a number of checklists and a trauma figure are present, where injuries can be marked. Originally it had two copies with carbon-paper technology, so that a copy could remain with the patient in the hospital. 


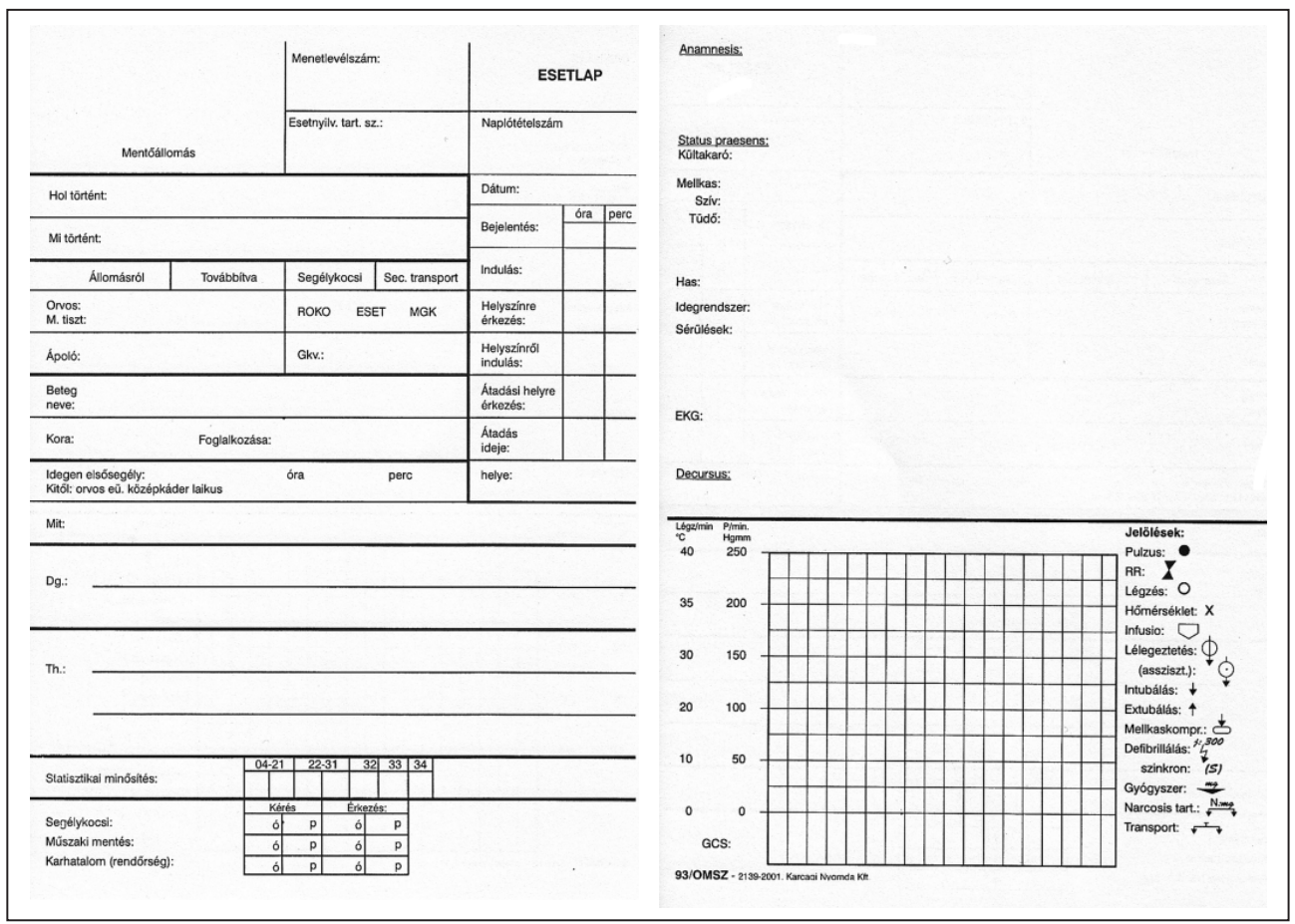

Figure 1. Prehospital patient report form of the National Ambulance Service

When a patient is handed over in a trauma center or emergency department in Hungary, some institutions require the filling out of their own A4 or A5 size patient registration forms, reporting the prehospital events, diagnosis and treatment. In other institutions parts of these data (what happened, when and where) is recorded by an administrator and additional more detailed verbal description is given to the doctor who admits the patient. Other hospitals require a narrative, they usually give a piece of blank A4 paper and the leader of the ambulance team writes about the what, when, where (happened) and about treatment.

The variety of documentation, and the multiple recording of patient data, especially physiological parameters and treatment raises a justifiable demand on the development of a uniform patient report form, that is both a prehospital documentation for ambulance services and contains enough and relevant information about the patient, the mechanism of injury (or illness) and the prehospital treatment for hospital admission. Data collection and statistics should also be included in the scheme in the long run, which is inherent to a well organized form, that has a number of checkboxes and selection lists.

Prehospital documentation has multiple overlapping components. Structured data content helps minimizing overlaps, support electronic data storage and processing, and last but not least helps on site providers by securing logical diagnostic sequences. That is why protocols were developed in accordance with these goals. An example of a Franco-German prehospital care system was chosen, because Hungarian prehospital care is organized along this scheme. In Germany DIVI (Deutsche Interdisziplinäre Vereinigung für Intensiv- und 


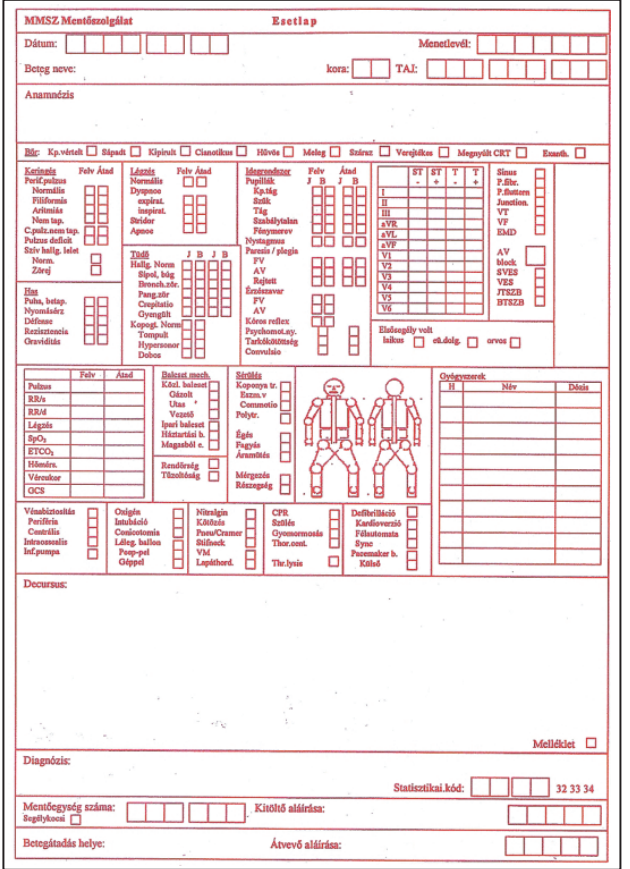

Figure 2. Prehospital patient report form of the Ambulance Service of the Hungarian Maltese Charity Service

Notfallmedizin, German Interdisciplinary Society for Intensive and Emergency Medicine) coordinated the development of the uniform national protocol for high-level ambulance units (the ones with a doctor or a college graduate paramedic) in 1991, and in 1994 it was followed by the protocol for basic level ambulance units. A large number of emergency services use these protocols from the beginning. Their success is proven by translation to various other languages. In 1994 version 4 , in 2000 version 4.2 came out, the latter did not cover structural changes, just optimized some details.

Documents, and patient report forms based on the DIVI protocol have a uniform data structure that is the result of collaboration of each professional bodies working in connection with prehospital care. First MIND (Minimale
Notarztdatensatz, Minimal Prehospital Care Data Set) appeared in 1996, followed by MIND2 in 2003. MIND's contents map the DIVI protocol, it has a structured format, and is suitable for each ambulance unit levels from basic to highest. Patient registration forms based on MIND2 are widely used (when necessary with local modifications), can be used as a transfer documentation and facilitate data recording on computers. DIVI has a patient report form, that uses MIND2 as its basis, however it contains slightly more data, than that MIND2 requires.

MIND2 is an XML-based (Extensible Markup Language), hierarchical text document, transparent to and editable by both humans and computers. Core data come from multiple sources (e.g. prehospital care providers, hospitals). Part of data covers circumstances of the incident (scene, etc.), ambulance characteristics and some information about the hospital. Patient and prehospital care data is covered in a timeline structure, utilizing scoring systems as often as possible. MEES (Mainz Emergency Evaluation Score) is used, which covers individually marked physiological parameters and other data (age, consciousness, GCS, systolic blood pressure, ECG, heart rate, respiratory rate, oxygen-saturation, pain according to the visual analog scale, blood sugar level, respiration characteristics, etc.). Diagnosis is listed according to organ groups. Trauma cases cover number and severity of injuries (monotrauma, multitrauma, polytrauma), type (blunt or penetrating) and location. Injury mechanism (fall from height, pedestrian, motor vehicle occupant, motorcyclist, bicyclist, other mechanism) and time of injury are covered too. Resuscitation data are in accordance with the current Utstein-template. Therapy can be chosen from the listed 30 interventions. Medications can be detailed in a timeline scheme. On-site and handover score values help evaluate patient's status and the effects of interventions during prehospital care. 
German company DokuFORM (http:// www.dokuform.de/) produces ambulance patient report forms in accordance with DIVI 4.2 (MIND2) both as mass product (Figure 3), and according to specific individual needs. There are traditional and modern paper-based versions, the latter to be filled with a digital pen to facilitate quick and exact data storage and transfer. DIVIDOK®-Online is a clientserver system to store these forms, that can be scanned or filled with a digital pen. During patient admission in the hospital a copy of the patient form is ready to be handed over together with the patient.

Theoretical and clinical experts of many fields collaborate in the ongoing knowledge base development of injury prevention and resuscitation. Their work resulted in the UtsteinStyle (or Utstein-template) for out-of-hospital resuscitations, in 1990. Utstein-Style contains uniform definitions, terminology and data sets. Utstein-Style is continuously revised and updated since then, and new areas have Utstein-Styles developed in the meantime (Pediatric Advanced Life Support, major trauma, drowning, etc.). The Utstein-Style for Major Trauma appeared in 1999, as the result of the extensive work of International Trauma Anaesthesia and Critical Care Society (ITACCS). This version covered prehospital and early hospital phases, previous illnesses and outcome prediction in an object-oriented model structure. In accordance with earlier Utsteintemplates this template has Core and Optional data variables. Model elements are divided into five categories, one about the healthcare network, one about the patient, data about treatment and outcome, as well as ethical aspects, documentation and methodology. Type, severity and mechanism of injury, the site where it happened are all recorded. Prehospital and in hospital provider data are similar to those in the resuscitation template.

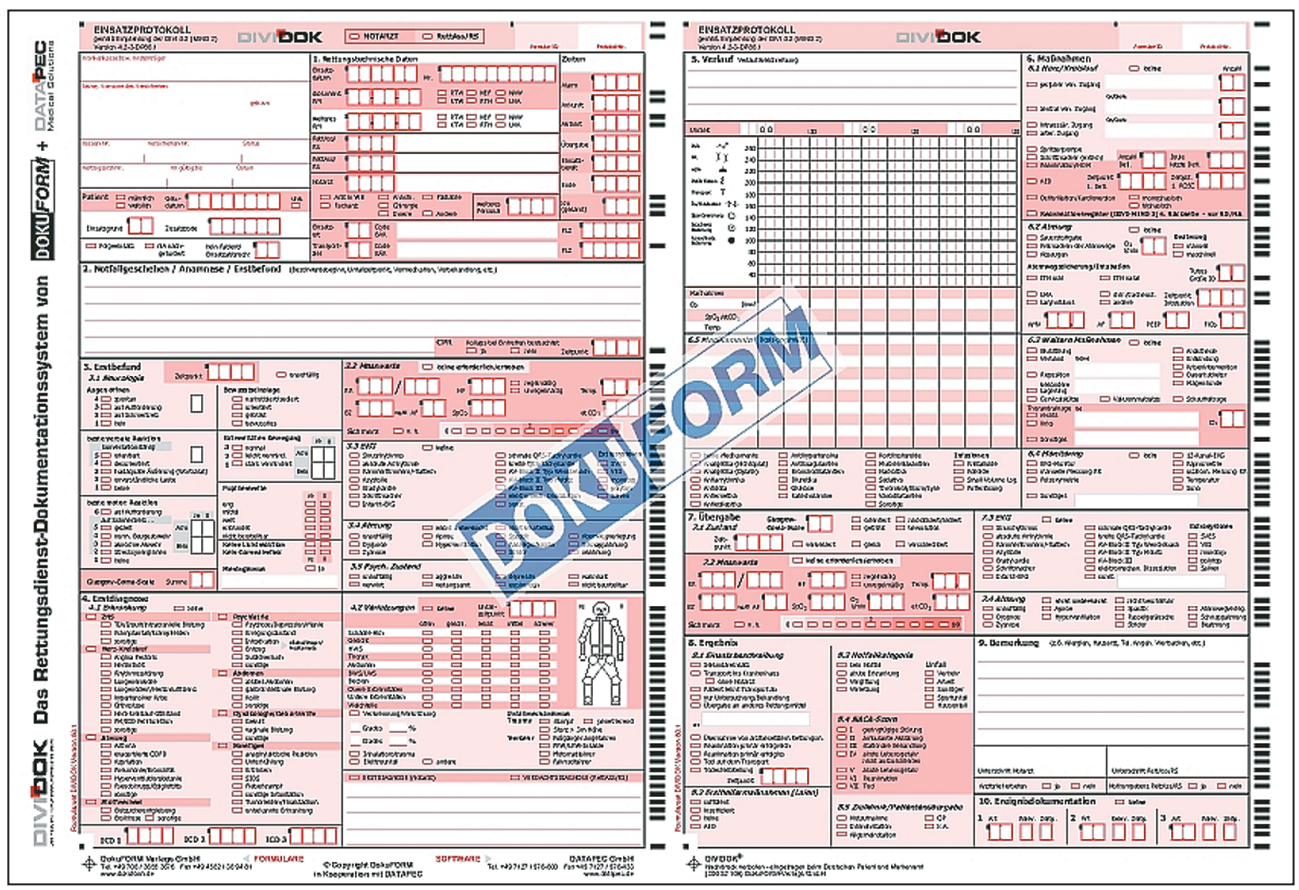

Figure 3. DokuFORM ambulance patient report form in accordance with DIVI 4.2 (MIND2) 
Patient data include age, gender, estimated height and weight, previous illnesses, physical status, results of prehospital examinations, treatment, and other categories when necessary (e.g. bicyclist, pedestrian, passenger of driver of a vehicle, etc.) Documentation and methodology chapter covers data collection techniques (e.g. GPS - Global Positioning System data for time and location). The purpose of the 1999 Utstein-Style for major trauma was data collection and statistical processing. This goal was not met, because there was no international consensus about application and applicability, besides the amount and nature of required data were to complicated. A revision followed in 2007 as a collaboration of some of the most important European Trauma organizations: Scandinavian Networking Group for Trauma and Emergency Management (SCANTEM), TARN (Trauma Audit \& Research Network) from the United Kingdom, the German Society for Trauma Surgery (Deutsche Gesellschaft für Unfallchirurgie, DGU) and their trauma register (DGU-TR), and the Italian National Register of Major Trauma (Registro Intraospedaliero Multiregionale Traumi Gravi, RITG). The revision resulted in only one inclusion criterion (New Injury Severity Score [NISS] value of 15 or more), 5 exclusion criteria, and 35 exactly specified and compulsory core data variables divided into 3 groups. Core data variables were chosen in accordance with the following considerations: they have to be unambiguous and easy to fill. Core data variable groups are Predictive models (23 variables), System Characteristic Descriptors (8 variables) and Process Mapping (4 variables).

National trauma registers are established in quite a number of countries worldwide, however the comparison is extremely difficult among their data. A typical trauma register is UK's TARN, which served as a model for the EuroTARN Group having 18 member states at the time of this writing (Austria, Belgium,
Bosnia-Hercegovina, Croatia, Denmark, Germany, Greece, Ireland, Italy, Macedonia, the Netherlands, Norway, Portugal, Slovakia, Spain, Sweden, Switzerland, and the United Kingdom). A 2007 EuroTARN study examined whether it is possible to compare European trauma registries. The result was positive, minimal additional infrastructure is needed to build a web-based system for this task. There is a wide support across Europe, to make comparisons of efficiency and outcome. The variety of national trauma registers urges unification, which leads to a uniform data set, coding rules, inclusion and exclusion criteria. UtsteinStyle is such a scheme. Its 2007 revision is not a final version, the system is continuously checked and updated when necessary.

\section{Conclusion}

According to literature review and the study of solutions form various countries, and our everyday practice in ambulance work and inhospital trauma care, we concluded that the new patient report form for Hungarian ambulance services should follow these aspects:

- logical setup, transparency, support for diagnostic steps

- support for scoring systems

- easy to fill with drawings and lists

- injury mechanisms are detailed

- road traffic accident specialties are drawn (a schematic car, where location of injured, collision sites, etc. can be marked)

- everything "compressed" into one document (single or multiple pages)

- paper-based version, with the support of electronic data processing

- a (carbon-) copy could be handed over with the patient in the hospital, or ideally can get to the hospital before the patient with an electronic communication system

- data content in accordance with reanimation and other specific registers 
- legal and ethical aspects fully covered

- equally emphasized documentation for trauma and illnesses (neurological, cardiological, etc.)

Data content has to be a consensus of many disciplines and professional bodies. It is important to study international examples and local circumstances. Patient report form has to cover the professional principles of prehospital care, with data relevant to examination and treatment methods and devices. Integration of protocols helps provide uniform high-level medical attendance, and ensures that no important diagnostic or treatment phase drops out.

\section{REFERENCES}

1. Andrianopoulos N. Moscow fuel. Traffic Technology International [Internet]. 2010 Jan; Forrás: http://viewer.zmags.com/publication/6e26c868\#/ $6 e 26 c 868 / 6$

2. Tscherne $H$, editor. Unfallchirurgie - Trauma Management. Springer, Heidelberg, 1997.

3. Gorove L, Szegeczky D, Szokoly M. Politraumatizáltak és súlyos sérültek helyszíni ellátása. In: Oxiológia. Medicina. 2006; pp. 511-526.

4. Butcher N, Balogh ZJ. The definition of polytrauma: the need for international consensus. Injury, Int. J. Care Injured [Internet]. 2009 Nov; 40(Supplement 4):S12-S22.

5. WHO | Road traffic injuries [Internet]. Forrás: http://www.who.int/violence_injury prevention/ road_trafficlen/

6. Dr. Márton E, Dr. Gorove L. A súlyos sérült kórházon kívüli ellátása. Az Országos Mentôszolgálat Módszertani útmutatója. 2008.

7. The Medical Algorithms projekt [Internet]. http://www.medal.org/

8. The Trauma Audit \& Research Network Procedures manual [Internet]. 2009 Sep; https:// www.tarn.ac.uk/content/downloads/53/ Full\%20Procedures\%20-\%20Sept\%202009.pdf

9. Lossius H. Erratum to "Reporting data following major trauma and analysing factors associated with outcome using the new Utstein style recommendations": [Resuscitation 50 (2001) 2632729]. Resuscitation [Internet]. 2004 11;63(2): 227.

10. Ringdal K, Coats T, Lefering R, Di Bartolomeo S, Steen $P$, Røise $O$ et al. The Utstein Template for Uniform Reporting of Data following Major Trauma. A joint revision by SCANTEM, TARN, DGU-TR, and RITG. Scand J Trauma Resusc Emerg Med [Internet]. 2008;16(7).

11. Brohi $K$. The Utstein template for uniform reporting of data following major trauma: A valuable tool for establishing a pan-European dataset. Scand J Trauma Resusc Emerg Med [Internet]. 2008;16(8).

12. Ringdal K, Coats $T$, Lefering $R$, Di Bartolomeo $S$, Steen $P$, Røise $O$, et al. The Utstein Trauma Template for Uniform Reporting of Data following Major Trauma: Data Dictionary. Version 1.1.1. [Internet]. 2009 May 19; Forrás: http://www.scantem.org/misc/pdf/Revised Utstein_Template___Data_Dictionary_ v1.1.1.pdf

13. The revised Utstein Template for Uniform Reporting of Data following Major Trauma: User manual (Version: 1.1).

14. Wolfgang F. Dick. Anglo-American vs. FrancoGerman Emergency Medical Services System. Prehospital and Disaster Medicine [Internet]. 2003;18(1):29-37. 
15. Probst C, Paffrath T, Krettek C, Pape HC. The German Trauma Registry. Comparative Update on Documentation of Trauma in Seven National Registries. Eur J Trauma [Internet]. 2006 8;32(4):357-364.

16. Confronting "Death on Wheels" Making Roads Safe in Europe and Central Asia [Internet]. Forrás: http://go.worldbank.org/DR6NO0BOP0

17. Vaca FE, Anderson CL, Herrera H, Patel C, Silman E, DeGuzman R et al. Crash Injury Prediction and Vehicle Damage Reporting by Paramedics: A Feasibility Study. WestJEM [Internet]. 2009 May 21;10(2):62-67.

18. Messelken M, Schlechtriemen T. Der minimale Notarztdatensatz MIND2. Notfall \& Rettungsmedizin [Internet]. 2003 May;6(3):189-192.

19. Lefering $R$. Development and validation of the revised injury severity classification score for severely injured patients. Eur J Trauma Emerg Surg [Internet]. 2009 9;35(5):437-447.

20. Moecke H, Dirks B, Friedrich H, Hennes H, Lackner $C$, Messelken $M$ et al. DIVI-Notarzteinsatzprotokoll, Version 4.2. Notfall \& Rettungsmedizin [Internet]. 2004 Jun;7(4):259-261.

21. Kuisma M, Väyrynen T, Hiltunen T, Porthan K, Aaltonen J. Effect of introduction of electronic patient reporting on the duration of ambulance calls. The American Journal of Emergency Medicine [Internet]. 2009 10;27(8):948-955.

22. Pohlman TH, Bjerke HS, Offner P. eMedicine Trauma: Trauma Scoring Systems [Internet]. eMedicine Trauma. 2009 Jul 23.

23. Johnson D, Wilson M. Improving prehospital information for trauma patients. Australian
Emergency Nursing Journal [Internet]. 1998 $10 ; 1(5): 12-13$.

24. Laudermilch DJ, Schiff MA, Nathens AB, Rosengart MR. Lack of Emergency Medical Services Documentation Is Associated with Poor Patient Outcomes: A Validation of Audit Filters for Prehospital Trauma Care. Journal of the American College of Surgeons [Internet]. 200912.

25. Reinhardt T, Hennes H. Mainz Emergency Evaluation Score (MEES). Notfall \& Rettungsmedizin [Internet]. 1999 Oct;2(6):380-381.

26. Make Roads Safe [Internet]. Forrás: http:// www. makeroadssafe.org/publications/ Documents/decade_of_action_report_lr.pdf

27. Bouamra O, WrotchfordA, Hollis S, VailA, Woodford $M$, Lecky $F$. Outcome prediction in trauma. Injury, Int. J. Care Injured [Internet]. 2006 Dec;37(12):1092-1097.

28. Jenkin A, Abelsonmitchell N, Cooper S. Patient handover: Time for a change? Accident and Emergency Nursing [Internet]. 2007 7;15(3): 141-147.

29. Gorove L, Szegeczky D, Szokoly M. Politraumatizáltak és súlyos sérültek helyszíni ellátása. In: Oxiológia. Medicina. 2006; p. 511-526.

30. Präklinische Erfassung der Verletzungsschwere aufgrund technischer Parameter am Unfallort. Notfall Rettungsmed [Internet]. 2007 6;10(5): 372-374.

31. Senkowski $C$. Trauma scoring systems: a review. Journal of the American College of Surgeons [Internet]. 1999 11;189(5):491-503.

\section{Petra Aradi}

Budapest University of Technology and Economics

Department of Mechatronics, Optics and Engineering Informatics

1111 Budapest, Múegyetem rkp. 3. 